\title{
The Perinatal Care Aims to Reduce Morbidity after Reducing Mortality in Japan
}

\section{Kazuo Maeda*}

Department of Obstetrics and Gynecology (Emeritus), Tottori University Medical School, Yonago, Japan

Maternal mortality was 410 in 100,000 births and neonatal mortality was 78 in 1,000 Live births, and estimated perinatal mortality after 28 weeks of pregnancy from maternal mortality was 135 in 1,000 births in 1899 when the first perinatal statistics was officially reported in Japan. In 2008, 110 years later, maternal mortality was 3.5 (1/117 of $1899)$, neonatal mortality was $1.2(1 / 65$ of 1899$)$, and actual perinatal mortality was 2.9 (1/47 of 1899$)$. The reduction velocity was slow in the first 50 years, while it was accelerated after 1950 in both maternal and neonatal mortality. Actual perinatal mortality was 47 in 1950 and 2.9 in 2008 (1/16 of 1950). The reason of the improvements would be the change of birth places, i.e. home delivery was usual before 1950, while the birth place quickly changed to hospitals and obstetric clinics, where hospital delivery was almost $100 \%$ after 1970 . The medicine and medical care would greatly contribute the reduction of maternal and perinatal mortalities [1].

The most interesting change was the definition of perinatal mortality in 1979, where national perinatal mortality was reported by the death after 22 weeks of pregnancy, while it was defined by the deaths after 28 weeks of pregnancy in 1950, that means the babies born before 28 gestational weeks were unable to survive in 1950, while survived preterm babies born at 22 weeks existed in 1979. In the recent cohort study, 36\% of babies born in 22 weeks survived at 3 years, $62.9 \%$ of babies born in 23 weeks were also survived at 3 years [2]. This marvelous change was achieved by the progress of neonatalogy and tremendous efforts of neonatologists in the NICU.

The reduction of mortalities in preterm babies is the prominent progress, of which care system would be excellent and further extend their favorable success in the future, while the morbidity would be decreased, and the subject will be the brain damages in every stage of pregnancy, delivery and postnatal lives. Despite the marvelous survival of preterm VLBW babies mentioned above [2], Cognitive delay developed in $57.1 \%$ of babies born in 22 gestational weeks at the evaluation in 3 years, $50 \%$ in the babies born in 23 weeks, and $32.2 \%$ of babies born in 24 weeks.

Although various reasons are present in preterm births, preterm labor contraction may be an important factor of preterm births, despite pharmaceutical tocolysis was performed in cases of preterm labor. As a new concept on the labor contraction, the regular contraction is a physiologic oscillation caused by the positive feed-back through the nerve between the uterus and brain [3]. It will be expected to suppress preterm uterine contraction by the sedation of nerve between the uterus and brain by using sedative or anesthetic technique, and prolong the pregnancy duration for some weeks, hopefully at longest to the full term delivery.

In another cause of brain damage of preterm babies, neonatal brain PVL followed by CP developed in $18 \%$ of neonates when the periventricular echo density (PVE) of the fetal brain persisted until the preterm birth, while there was neither PVL nor CP if the PVE disappeared before preterm birth, or in full term birth [4]. Although $\mathrm{MgSO}_{4}$ administration to the preterm labor with PVE, hydrocortisone or erythropoietine therapy to the preterm neonate who has PVE in the brain are considered, while another strategy will be the prolongation of pregnancy to the full-term birth by the suppression of preterm labor contraction.

The FHR acceleration, physiological sinusoidal FHR and FHR baseline variability would develop by the stimulation of fetal movements to the mid-brain. The variability would be caused by minor fetal movements, which was visible after the augmentation of fetal movement trace in the actocardiogram. The loss of variability has been rare, but it would be the sign of severe fetal brain damage possibly followed by CP. The author recommend C-section before the loss of variability, when the author's hypoxia index was as large as 20-24, instead of the C-section after recognition of the loss of variability [5].

\section{References}

1. Kamitani K (2011) Maternal and Child Health Statistics of Japan. Boshi Hoken Jigyodan, Tokyo

2. Ishii N, Kono Y, Yonemoto N, Kusuda S, Fuzimura M (2014) Outocomes of infants born at 22 and 23 weeks' gestaion 1-10.

3. Maeda K (2013) Uterine contractions in normal labor developed by a positive feed-back and oscillation. J Health Med Inform 4: 130.

4. Maeda K (2013) A proposal to reduce congenital cerebral palsy. J Health Med Inform 4: 135

5. Maeda K (2012) Actocardiographic analysis of fetal hypoxia detected by the bradycardia, loss of fetal heart rate acceleration and long term variability. J Health Med Inform 4: 118.
*Corresponding author: Kazuo Maeda, Department of Obstetrics and Gynecology (Emeritus), Tottori University Medical School, Yonago, Japan, Tel: 81-859-22-6856; E-mail: maedak@mocha.ocn.ne.jp

Received August 08, 2014; Accepted August 11, 2014; Published August 16 2014

Citation: Maeda K (2014) The Perinatal Care Aims to Reduce Morbidity after Reducing Mortality in Japan. J Health Med Informat. 5: e132. doi:10.4172/2157$7420.1000 \mathrm{e} 132$

Copyright: (c) 2014 Maeda K. This is an open-access article distributed under the terms of the Creative Commons Attribution License, which permits unrestricted use, distribution, and reproduction in any medium, provided the original author and source are credited. 